\title{
Category norms for verbal items in 25 categories for children in Grades 2-6
}

\author{
CARLA J. POSNANSKY \\ University of Rochester, Rochester, New York 14627
}

\begin{abstract}
Three hundred and seventy-five children in Grades 2, 3, 4, and 6 were asked to generate instances of 25 different categories within a time period of 1 min per category. Data were tallied so that category instances are ranked as to proportion of subjects making each response at each grade level. Indications of the average number of category instances generated by children at each grade level within each category are provided.
\end{abstract}

In 1969, Battig and Montague published normative category information for 56 categories with adult subjects. Developmental studies of organization of memory have been dependent upon this adult normative information, with the exception of Nelson $(1968,1969)$, without regard to whether the use of the same normative information is appropriate. The developmental manipulation of type of list structure is, however, no doubt best based upon normative information that has also been collected developmentally.

\section{METHOD}

In the data collection, 375 children in Grades $2,3,4$, and 6 served as subjects. The children were students at Vanderhoof Elementary School in Arvada, Colorado (82\%), or members of Mile Hi Girl Scout groups in Boulder, Colorado (18\%). The number of subjects in each grade (by sex) is shown in Table 1. Subjects may be generally described as members of the upper middle-class socioeconomic stratum. Data collection lasted $25 \mathrm{~min}$ after an initial instructional period. Instructions to the subjects were as follows: "We are going to play a word game. This is not a test. We are going to think of different kinds of things. That is, I am going to tell you a thing and you tell me all different kinds of it that you can think of. I might say, for example, 'musical instruments' and you could answer 'violin' and 'piano' and 'guitar.' You can tell me as many things as you can think of. If I said 'names' you could say 'Jane' and 'Tom' and 'Sally' and as many others as you think of. Now, if I said 'things that you study in school' what kinds of things would you say?" (Pause for verbal responses.)

"Now I want you to write down the things you can think of. Do not say any things aloud. Spelling will not count. Remember spelling will NOT count-just spell the best that you

This paper was prepared while the author was supported by a National Science Foundation predoctoral fellowship. Partial support was provided by National Science Foundation Grant GB 34077X. The author is now at the University of Rochester, Center for Development, Learning, and Instruction, Rochester, New York 14627. Thanks are due to Ken Vendena, Principal, and the staff of Vanderhoof Elementary School in Arvada, Colorado, for their cooperation, as well as to Audrey House of the Mile Hi Girl Scout Council and to the Boulder Valley Girl Scout leaders. The author appreciates the advice and assistance of William F. Battig, Linda S. Gleason, James W. Pellegrino, and an anonymous reviewer. can. When I tell you what to think about, you can start to write in your booklet, and, when I tell you to stop, we will turn the page and I will give you a new thing to think and write about. You do not have to write down what I say, only what you can think of.

"Do you have any questions?"

Subjects were provided with a 25-page test booklet and were allowed $1 \mathrm{~min}$ to write after each category was announced by the experimenter. After each "stop" command, subjects were asked to turn to a new page in the booklet, and the next category was announced immediately.

Twenty-five categories from the Battig and Montague (1969) norms were selected because the experimenter judged them to be easily explainable to children. In some instances, phrases rather than single words designated categories. The reader is referred to the list of categories shown in Table 2.

Subjects were run in groups ranging in size from 2 to 45 children.

\section{RESULTS}

All legible words were tallied separately for each category and each grade level. The basic data are presented in the Appendix, in terms of the proportion of subjects in each grade who made the specific response being considered. All instances of a category with a frequency of two or more are included. Singular and plural responses naming the same exemplar were combined, except where the frequencies of both forms were each substantial in their own right. Responses were not separated according to grammatical form or spelling, except in cases wherein the frequency of each of two or more different forms was greater than 10 . The mean number of distinct responses to each category is provided following each category listing.

Table 2 provides information regarding the number of

Table 1

Number of Subjects in Each Grade (by Sex)

\begin{tabular}{cccc}
\hline Grade & N & Female & Male \\
\hline 2 & 81 & 45 & 36 \\
3 & 64 & 40 & 24 \\
4 & 126 & 65 & 61 \\
6 & 104 & 60 & 44 \\
\hline
\end{tabular}


Table 2

Number of Distinct Instances Generated per Category

\begin{tabular}{|c|c|c|c|c|c|}
\hline \multirow[b]{2}{*}{ Category } & \multicolumn{4}{|c|}{ Grade } & \multirow[b]{2}{*}{ Mear } \\
\hline & $\begin{array}{c}2 \\
(n=81)\end{array}$ & $\begin{array}{c}3 \\
(n=64)\end{array}$ & $\begin{array}{c}4 \\
(n=126)\end{array}$ & $\begin{array}{c}6 \\
(n=104)\end{array}$ & \\
\hline 1. Fruits & 26 & 24 & 27 & 34 & 28.0 \\
\hline 2. Birds & 47 & 63 & 53 & 67 & 57.5 \\
\hline 3. Carpenter's Tools & 36 & 60 & 38 & 66 & 50.0 \\
\hline 4. Parts of the Body & 47 & 64 & 65 & 70 & 61.5 \\
\hline 5. Parts of a Building & 81 & 110 & 98 & 123 & 103.0 \\
\hline 6. Weather Phenomena & 45 & 72 & 52 & 77 & 61.5 \\
\hline 7. Places One Can Stay Away From Home & 49 & 73 & 67 & 76 & 66.3 \\
\hline 8. Kitchen Utensils & 68 & 90 & 94 & 99 & 87.8 \\
\hline 9. Vegetables & 36 & 49 & 44 & 42 & 42.8 \\
\hline 10. Sports & 43 & 116 & 76 & 83 & 79.5 \\
\hline 11. Denominations of Money & 9 & 21 & 22 & 25 & 19.3 \\
\hline 12. Footwear & 30 & 75 & 49 & 70 & 56.0 \\
\hline 13. Toys & 81 & 146 & 105 & 142 & 118.5 \\
\hline 14. Trees & 46 & 75 & 51 & 70 & 60.5 \\
\hline 15. Clothing & 45 & 64 & 60 & 72 & 60.3 \\
\hline 16. Relatives & 43 & 58 & 28 & 43 & 43.0 \\
\hline 17. Furniture & 35 & 53 & 70 & 71 & 57.3 \\
\hline 18. Crimes & 44 & 54 & 51 & 73 & 55.5 \\
\hline 19. Flowers & 38 & 73 & 39 & 55 & 51.3 \\
\hline 20. Animals & 61 & 75 & 69 & 84 & 72.3 \\
\hline 21. Insects & 36 & 45 & 34 & 58 & 43.3 \\
\hline 22. Colors & 29 & 46 & 30 & 45 & 37.5 \\
\hline 23. Vehicles and Means of Transportation & 52 & 77 & 58 & 83 & 67.5 \\
\hline 24. Ways to Measure Distance & 22 & 58 & 38 & 55 & 43.3 \\
\hline 25. Ways to Measure Time & 28 & 54 & 28 & 58 & 42.0 \\
\hline Mean & 43.08 & 67.84 & 53.84 & 69.64 & \\
\hline
\end{tabular}

distinct instances generated by subjects at a given grade level for a particular category. Means for each grade and category are provided.

\section{REFERENCES}

Batrig, W. F., \& Montague, W. E. Category norms for verbal items in 56 categories: A replication and extension of the Connecticut category norms. Journal of Experimental Psychology Monograph, 1969, 80, 1-46.

Nelson, K. J. Developmental factors in the organization of free recall. Unpublished doctoral dissertation, University of California, Los Angeles, 1968.

NeLson, K. J. The organization of free recall by young children. Journal of Experimental Child Psychology, 1969, 8, 284-295.

\section{APPENDIX}

Listed below are the proportions of children (Grades 2-6) generating instances of the 25 different categories. The mean number of instances is presented at the bottom of each category for each grade.

\begin{tabular}{lccccc}
\multicolumn{5}{c}{$\begin{array}{c}\text { Category 1 } \\
\text { Fruits }\end{array}$} \\
& \multicolumn{5}{c}{ Grade } \\
\cline { 2 - 6 } Instance & 2 & 3 & 4 & 6 \\
\hline apple & .765 & .933 & .897 & .933 \\
orange & .519 & .859 & .794 & .904
\end{tabular}

Category 1 Continued

\begin{tabular}{lcccc}
\hline & \multicolumn{3}{c}{ Grade } \\
\cline { 2 - 5 } \multicolumn{1}{l}{ Instance } & 2 & 3 & 4 & 6 \\
\hline grape & .358 & .500 & .516 & .635 \\
banana & .346 & .547 & .611 & .798 \\
pear & .259 & .453 & .603 & .596 \\
peach & .148 & .453 & .437 & .423 \\
pineapple & .123 & .109 & .214 & .308 \\
grapefruit & .074 & .141 & .151 & .250 \\
lemon & .062 & .141 & .095 & .192 \\
cherry & .049 & .203 & .222 & .221 \\
tomato & .049 & & .016 & .096 \\
plum & .037 & .141 & .230 & .250 \\
coconut & .025 & & .024 & .048 \\
lime & .025 & .078 & .087 & .154 \\
tangerine & .025 & .031 & & .096 \\
cantaloupe & & .156 & & .038 \\
watermelon & & .063 & .056 & .058 \\
apricot & & .047 & .071 & .135 \\
prune & & .047 & .048 & .144 \\
strawberry & & .047 & .040 & .192 \\
blackberry & & & .040 & .029 \\
blueberry & & & .032 & .029 \\
raspberry & & & .016 & .077 \\
papaya & & & & .029 \\
avocado & & & & .019 \\
fig & & & .019 \\
pomegranate & & & & .019 \\
Mean & & & & 6.76 \\
\hline & & & & \\
\hline
\end{tabular}


Category 2 Birds

\begin{tabular}{|c|c|c|c|c|c|c|c|c|c|}
\hline \multirow{3}{*}{ Instance } & & \\
\hline & \multicolumn{4}{|c|}{ Grade } & \multirow[b]{2}{*}{ Instance } & \multicolumn{4}{|c|}{ Grade } \\
\hline & 2 & 3 & 4 & 6 & & 2 & 3 & 4 & 6 \\
\hline bluebird & .432 & .406 & .429 & .317 & nightingale & & & & .019 \\
\hline robin & .284 & .672 & .659 & .673 & oriole & & & & .019 \\
\hline bluejay & .185 & $\begin{array}{r}.406 \\
359\end{array}$ & .262 & $\begin{array}{r}.346 \\
144\end{array}$ & Mean & 2.73 & 4.94 & 4.21 & 5.43 \\
\hline $\begin{array}{l}\text { parrot } \\
\text { eagle }\end{array}$ & $\begin{array}{l}.185 \\
.173\end{array}$ & $\begin{array}{l}.359 \\
.281\end{array}$ & $\begin{array}{l}.111 \\
.294\end{array}$ & $\begin{array}{l}.144 \\
.240\end{array}$ & \multirow{7}{*}{\multicolumn{5}{|c|}{$\begin{array}{l}\text { *The ptarmigan is the state bird of Alaska. A substantial number } \\
\text { of second graders had just completed a social studies unit on } \\
\text { Alaska, and this undoubtedly accounts for the high frequency of } \\
\text { this response. } \\
\text { Category } 3 \\
\text { Carpenter's Tools }\end{array}$}} \\
\hline sparrow & .148 & .313 & .254 & .510 & & & & & \\
\hline blackbird & .136 & .141 & .230 & .163 & & & & & \\
\hline crow & .111 & .094 & .206 & .202 & & & & & \\
\hline hummingbird & .111 & .156 & .119 & .144 & & & & & \\
\hline duck & .099 & .094 & .167 & .135 & & & & & \\
\hline ptarmigan* & .074 & & & & & & & & \\
\hline $\begin{array}{l}\text { woodpecker } \\
\text { hawk }\end{array}$ & $\begin{array}{l}.062 \\
.049\end{array}$ & $\begin{array}{l}.047 \\
078\end{array}$ & $\begin{array}{r}.071 \\
397\end{array}$ & $\begin{array}{r}.183 \\
144\end{array}$ & & \multicolumn{4}{|c|}{ Grade } \\
\hline pigeon & .049 & .109 & & .115 & Instance & 2 & 3 & 4 & 6 \\
\hline bird & .037 & .141 & 056 & .154 & hammer & .840 & .891 & .944 & .981 \\
\hline $\begin{array}{l}\text { canary } \\
\text { ostrich }\end{array}$ & $\begin{array}{l}.037 \\
.037\end{array}$ & .078 & .016 & .038 & saw & .432 & .609 & .675 & .702 \\
\hline parakeet & .037 & .094 & & .067 & screwdriver & .383 & .547 & .643 & .712 \\
\hline redbird & .037 & .031 & .016 & & wrench & .346 & .359 & .563 & .413 \\
\hline swan & .037 & .031 & .056 & .019 & nail & .309 & .594 & .476 & .625 \\
\hline goose & .025 & & .048 & .087 & screw & .160 & .141 & .183 & .260 \\
\hline owl & .025 & .031 & .056 & .048 & pliers & .099 & .297 & .111 & .183 \\
\hline peacock & .025 & & .032 & .067 & drill & .037 & .219 & .190 & .231 \\
\hline redbreast & .025 & .047 & & & bolt & .025 & .063 & .048 & .067 \\
\hline roadrunner & .025 & & & .019 & glue & .025 & & & .019 \\
\hline rooster & .025 & & & & plane & .025 & & & .048 \\
\hline starling & .025 & & & .019 & wood & .025 & .078 & .056 & .048 \\
\hline nest & & .156 & & & chisel & & .063 & & .038 \\
\hline chicken & & .094 & .024 & .115 & shovel & & .063 & & \\
\hline eggs & & .078 & & & tools & & .063 & & \\
\hline mockingbird & & .063 & & .029 & board & & .047 & .071 & \\
\hline swallow & & .063 & & .029 & nut & & .047 & .040 & .058 \\
\hline tree & & .063 & & & cement & & .031 & & \\
\hline seagull & & .047 & & .048 & concrete & & .031 & & \\
\hline bald eagle & & .031 & & & hacksaw & & .031 & & .019 \\
\hline finch & & .031 & & .019 & jackhammer & & .031 & & \\
\hline flamingo & & .031 & .032 & .067 & knife & & .031 & .024 & .029 \\
\hline fly & & .031 & & & monkey wrench & & .031 & .024 & .019 \\
\hline hen & & .031 & & & ruler & & .031 & & .087 \\
\hline pelican & & .031 & .016 & & sandpaper & & .031 & & \\
\hline penguin & & .031 & & .038 & wire & & .031 & .016 & \\
\hline pheasant & & .031 & .040 & .077 & wirecutter & & .031 & & .019 \\
\hline quail & & .031 & .016 & .048 & work & & .031 & & \\
\hline redwing & & .031 & & & yardstick & & .031 & & \\
\hline turkey & & .931 & .071 & & axe & & & .079 & .019 \\
\hline wings & & .031 & & & blowtorch & & & .016 & \\
\hline wotm & & .031 & & & sander & & & .016 & .029 \\
\hline magpie & & & .079 & .106 & sledgehammer & & & .016 & \\
\hline cardinal & & & .063 & .173 & level & & & & .067 \\
\hline falcon & & & .048 & .038 & square & & & & .038 \\
\hline jay & & & .048 & .125 & file & & & & .029 \\
\hline dove & & & .040 & .067 & point & & & & .019 \\
\hline blue & & & .032 & & washer & & & & .019 \\
\hline vulture & & & .024 & .019 & Mean & 3.00 & 4.95 & 4.35 & 5.18 \\
\hline $\begin{array}{l}\text { bat } \\
\text { chick }\end{array}$ & & & $\begin{array}{l}.016 \\
.016\end{array}$ & & \multirow{3}{*}{\multicolumn{5}{|c|}{$\begin{array}{c}\text { Category } 4 \\
\text { Parts of the Body }\end{array}$}} \\
\hline meadowlark & & & .016 & .087 & & & & & \\
\hline scarecrow & & & .016 & & & & & & \\
\hline $\begin{array}{l}\text { lark } \\
\text { wren }\end{array}$ & & & & $\begin{array}{l}.096 \\
.067\end{array}$ & & \multicolumn{4}{|c|}{ Grade } \\
\hline $\begin{array}{l}\text { gull } \\
\text { chickadee }\end{array}$ & & & & $\begin{array}{l}.038 \\
029\end{array}$ & Instance & 2 & 3 & 4 & 6 \\
\hline flicker & & & & $\begin{array}{l}.029 \\
.029\end{array}$ & leg & .630 & .672 & .714 & .894 \\
\hline stork & & & & .029 & head & .556 & .688 & .659 & .731 \\
\hline
\end{tabular}


Category 4 Continued

\begin{tabular}{llllll} 
& \multicolumn{5}{c}{ Grade } \\
\cline { 2 - 6 } Instance & 2 & 3 & 4 & 6
\end{tabular}

\section{arm}

eye

foot

nose

ear

feet

toe

neck

hair

finger

mouth

bone

back

stomach

knee

face

body

brain

chest

elbow

heart

hip

ankle

blood

chin

muscle

rib

shoulder

thigh

tooth

tongue

bottom

butt

teeth

skin

toenail

belly button

breast

eyebrow

kidney

lip

liver

skull

wrist

lung

pelvis

intestines

jaw

nail

freckles

rear end

shin

thumb

fanny

backbone

appendix

calf

heel

pancreas

vein

Mean

.594

2

.568

.358

.296

.284

.222

.185

.185

.185

.173

.123

.111

.111

.099

.086

.086

.074

.062

.049

.049

.049

.049

.049

.049

.037

.037

.037

.025

.025

.025

.025

.025

3

$.625 \quad .706$

.422

.594

.344

.500

.250

.422

.172

.344

.438

.422

.063

.078

.172

.203

.047

.031

.109

.047

.109

.203

.141

.047

.047

.031

.063

.078

.078

.078

.063

.063

.063

.047

.047

.031

.031

.031

.031

.031

.031

$\begin{array}{ll}.111 & .019 \\ .119 & .125 \\ .048 & .029\end{array}$

$.016 \quad .058$

.024

.071

.016

.024

.016

.040

.024

.103

.095

.048

.048

.024

.024

.024

.016

.016

.016

.016

.058
.096
.048
.106
.019
.029

.837

.837
.471

.615

.538
.625

.471

.827

654
115

.212

.635

.452

.058
038

.038
.202

.240

.087

.077

.192

.173

.058
.144

dining room
glass

rug

stair

basement

chair

downstairs

hall

heater

light

metal

porch

steel

upstairs

table

garage

sink

.019 bathtub

clock

.058 cafeteria

.106 couch

.038

.019

.019

lamp

map

shelf

toilet

book

bulletin board

carpet

chalkboard

flag

.029

.048

.029

.019

.019

.019

.019

.019

gym

office

phone

playroom

school

stove

television

workroom

10.70

nail

Category 5

Parts of a Building

Grade

\section{Grade}

\begin{tabular}{llll}
\hline 2 & 3 & 4 & 6
\end{tabular}

$\begin{array}{llll}.556 & .359 & .714 & .567 \\ .333 & .375 & .548 & .471 \\ .284 & .266 & .397 & .317 \\ .198 & .281 & .103 & .260 \\ .185 & .297 & .381 & .519 \\ .136 & .250 & .095 & .115 \\ .136 & .172 & .333 & .173 \\ .123 & .234 & .079 & .038 \\ .123 & .203 & .262 & .558 \\ .123 & .219 & .508 & .365 \\ .086 & .219 & .016 & .106 \\ .062 & .094 & .063 & .038 \\ .062 & .031 & & .019 \\ .062 & .203 & .024 & .048 \\ .062 & .031 & .079 & .038 \\ .049 & & & \\ .049 & .047 & .103 & .096 \\ .049 & .047 & & .135 \\ .037 & & .032 & .019 \\ .037 & & .119 & .240 \\ .037 & .047 & .159 & .019 \\ .037 & .063 & & .029 \\ .037 & .172 & .024 & .077 \\ .037 & .031 & & .038 \\ .037 & & .040 & .087 \\ .037 & .031 & .063 & \\ .037 & .094 & & \end{array}$

.037

.025

.025

.025

.025

.025

.025

.025

.025

.025

.094

.094

.087

.135

.048

.203

.031

.031

.078

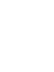

.067

.038

$.071 \quad .144$

$.016 \quad .029$

$.063 \quad .048$

.156
.094

.094
.094

.063

.063

.047

.047

.047

.047

.047

.047

.031

.031

.031

.031

.031

.031

.031

.031

.031

.031

.031

.031

.079

.032

.048

.016

.077

.032

.024

.019 
Category 5 Continued

\begin{tabular}{|c|c|c|c|c|}
\hline \multirow[b]{2}{*}{ Instance } & \multicolumn{4}{|c|}{ Grade } \\
\hline & 2 & 3 & 4 & 6 \\
\hline paint & & & .048 & \\
\hline step & & & .048 & \\
\hline chairs & & & .032 & \\
\hline plaster & & & .032 & .029 \\
\hline cabinet & & & .024 & .029 \\
\hline elevator & & & .024 & .077 \\
\hline den & & & .016 & \\
\hline drapes & & & .016 & \\
\hline dresser & & & .016 & \\
\hline furniture & & & .016 & \\
\hline screw & & & .016 & \\
\hline wallpaper & & & .016 & \\
\hline frame & & & & .048 \\
\hline library & & & & .048 \\
\hline rest room & & & & .048 \\
\hline rooms & & & & .038 \\
\hline fire escape & & & & .029 \\
\hline air conditioner & & & & .019 \\
\hline attic & & & & .019 \\
\hline classroom & & & & .019 \\
\hline curtain & & & & .019 \\
\hline foundation & & & & .019 \\
\hline furnace & & & & .019 \\
\hline hallway & & & & .019 \\
\hline shingles & & & & .019 \\
\hline window sill & & & & .019 \\
\hline wire & & & & .019 \\
\hline \multirow[t]{3}{*}{ Mean } & 3.81 & 6.23 & 5.45 & 6.62 \\
\hline & \multicolumn{3}{|c|}{$\begin{array}{c}\text { Category } 6 \\
\text { Weather Phenomena }\end{array}$} & \\
\hline & \multicolumn{4}{|c|}{ Grade } \\
\hline Instance & 2 & 3 & 4 & 6 \\
\hline snow & .728 & .750 & .849 & .827 \\
\hline rain & .593 & .719 & .856 & .779 \\
\hline sun & .383 & .172 & .405 & .346 \\
\hline hail & .259 & .500 & .381 & .462 \\
\hline hot & .210 & .344 & .294 & .356 \\
\hline cold & .198 & .313 & .310 & .394 \\
\hline sunny & .198 & .281 & .103 & .173 \\
\hline wind & .173 & .172 & .325 & .260 \\
\hline winter & .160 & 141 & .111 & .048 \\
\hline summer & .148 & .141 & .103 & .038 \\
\hline cloudy & .123 & .219 & .230 & .279 \\
\hline fog & .123 & .188 & .302 & .212 \\
\hline spring & .111 & .109 & .135 & .038 \\
\hline warm & .099 & .125 & .167 & .173 \\
\hline rainy & .086 & .094 & .024 & .125 \\
\hline fall & .074 & .125 & .125 & .029 \\
\hline snowy & .074 & .063 & & .115 \\
\hline sleet & .049 & .156 & .095 & .260 \\
\hline windy & .049 & .234 & .095 & .125 \\
\hline stormy & .037 & .094 & .071 & .048 \\
\hline tornado & .037 & .219 & .016 & .087 \\
\hline autumn & .025 & & & \\
\hline cool & .025 & .094 & .040 & .087 \\
\hline chilly & .025 & & & .019 \\
\hline hurricane & & .109 & .016 & .067 \\
\hline storm & & .109 & .111 & .038 \\
\hline foggy & & .094 & & .067 \\
\hline tidal wave & & .094 & & \\
\hline
\end{tabular}

Category 6 Continued

\begin{tabular}{|c|c|c|c|c|}
\hline \multirow[b]{2}{*}{ Instance } & \multicolumn{4}{|c|}{ Grade } \\
\hline & 2 & 3 & 4 & 6 \\
\hline lightning & & .078 & .016 & .019 \\
\hline thunder & & .078 & & .038 \\
\hline blizzard & & .063 & .079 & .048 \\
\hline flood & & .063 & & .038 \\
\hline freezing & & .063 & & .029 \\
\hline nice & & .063 & & \\
\hline dry & & .047 & .016 & .087 \\
\hline frost & & .047 & .167 & .077 \\
\hline sunshine & & .047 & .040 & .048 \\
\hline bad & & .031 & & \\
\hline damp & & .031 & & .038 \\
\hline freeze & & .031 & .016 & .029 \\
\hline good & & .031 & & \\
\hline ice & & .031 & .024 & \\
\hline smog & & .031 & .040 & .019 \\
\hline steaming & & .031 & & \\
\hline wet & & .031 & & .058 \\
\hline cloud & & & .183 & .029 \\
\hline dew & & & .040 & .029 \\
\hline fair & & & .040 & .019 \\
\hline dusty & & & .040 & \\
\hline humid & & & & .077 \\
\hline mild & & & & .038 \\
\hline misty & & & & .038 \\
\hline dust & & & & .029 \\
\hline earthquake & & & & .029 \\
\hline heat & & & & .029 \\
\hline cyclone & & & & .019 \\
\hline drought & & & & .019 \\
\hline gloomy & & & & .019 \\
\hline Mediterranean & & & & .019 \\
\hline mist & & & & .019 \\
\hline monsoon & & & & .019 \\
\hline Mean & 4.26 & 6.92 & 5.98 & 6.64 \\
\hline
\end{tabular}

Category 7

Maces One Can Stay Away from Home (Dwellings)

\begin{tabular}{|c|c|c|c|c|}
\hline \multirow[b]{2}{*}{ Instance } & \multicolumn{4}{|c|}{ Grade } \\
\hline & 2 & 3 & 4 & 6 \\
\hline hotel & .469 & .672 & .810 & .942 \\
\hline motel & .469 & .641 & .579 & .865 \\
\hline hospital & .444 & .703 & .730 & .587 \\
\hline house & .333 & .609 & .206 & .250 \\
\hline tent & .185 & .156 & .214 & .413 \\
\hline apartment & .148 & .328 & .135 & .144 \\
\hline home & .148 & .188 & & .077 \\
\hline trailer & .099 & .109 & .111 & .279 \\
\hline cabin & .074 & .063 & .103 & .135 \\
\hline car & .074 & .094 & .175 & .125 \\
\hline friends & .074 & & .079 & .077 \\
\hline camper & .062 & .094 & .167 & .279 \\
\hline grandma's & .062 & & & \\
\hline bedroom & .049 & .031 & & \\
\hline school & .049 & .203 & .048 & .029 \\
\hline barn & .025 & & & \\
\hline bath & .025 & & & \\
\hline bed & .025 & .047 & .016 & .019 \\
\hline building & .025 & .047 & & \\
\hline shack & .025 & & & .038 \\
\hline California & & .047 & & \\
\hline camp & & .047 & .087 & .058 \\
\hline
\end{tabular}


Category 7 Continued

\begin{tabular}{|c|c|c|c|c|}
\hline \multirow[b]{2}{*}{ Instance } & \multicolumn{4}{|c|}{ Grade } \\
\hline & 2 & 3 & 4 & 6 \\
\hline doctor & & .047 & & \\
\hline plane & & .047 & .016 & .029 \\
\hline rest home & & .047 & & .029 \\
\hline restaurant & & .047 & .016 & \\
\hline Boulder & & .031 & & \\
\hline campground & & .031 & .048 & .029 \\
\hline cottage & & .031 & & \\
\hline inn & & .031 & .024 & .077 \\
\hline lodge & & .031 & .024 & .115 \\
\hline room & & .031 & & .019 \\
\hline train & & .031 & .032 & .067 \\
\hline Winnebago & & .031 & & \\
\hline mountain & & & .111 & .019 \\
\hline camping & & & .048 & \\
\hline bus & & & .040 & \\
\hline town & & & .040 & \\
\hline city & & & .032 & \\
\hline neighbors & & & .024 & \\
\hline outside & & & .024 & \\
\hline airplane & & & .016 & .019 \\
\hline Alaska & & & .016 & \\
\hline boat & & & .016 & .019 \\
\hline cave & & & .016 & .048 \\
\hline church & & & .016 & \\
\hline lake & & & .016 & \\
\hline relative & & & .016 & \\
\hline truck & & & .016 & \\
\hline woods & & & .016 & .019 \\
\hline boarding house & & & & .038 \\
\hline $\begin{array}{l}\text { dorm } \\
\text { fort }\end{array}$ & & & & .038 \\
\hline $\begin{array}{l}\text { tort } \\
\text { gas station }\end{array}$ & & & & .019 \\
\hline tavern & & & & .019 \\
\hline Mean & 3.23 & 5.17 & 4.37 & 4.01 \\
\hline
\end{tabular}

Category 8

Kitchen Utensils

\begin{tabular}{lcccc}
\hline & \multicolumn{4}{c}{ Grade } \\
\cline { 2 - 5 } \multicolumn{1}{c}{ Instance } & 2 & 3 & 4 & 6 \\
\hline pan & .593 & .516 & .437 & .519 \\
spoon & .506 & .797 & .794 & .827 \\
knife & .444 & .672 & .770 & .788 \\
fork & .395 & .766 & .722 & .769 \\
stove & .272 & .359 & .159 & .317 \\
pot & .148 & .219 & .262 & .240 \\
bowl & .099 & .156 & .206 & .183 \\
oven & .086 & .109 & .143 & .144 \\
dish & .074 & .141 & .095 & .058 \\
mixer & .062 & .156 & .135 & .202 \\
plate & .049 & .250 & .175 & .173 \\
recipe & .049 & & & \\
refrigerator & .049 & .203 & .079 & .144 \\
food & .037 & & .024 & .019 \\
beater & .037 & .031 & .056 & .144 \\
blender & .025 & .094 & .127 & .173 \\
can opener & .025 & .141 & .024 & .135 \\
cookbook & .025 & & & \\
cookie & .025 & & & \\
egg beater & .025 & & .024 & .058 \\
freezer & .025 & & .016 & \\
glass & .025 & .078 & .087 & .058 \\
& & & &
\end{tabular}

Category 8 Continued

\begin{tabular}{|c|c|c|c|c|}
\hline \multirow[b]{2}{*}{ Instance } & \multicolumn{4}{|c|}{ Grade } \\
\hline & 2 & 3 & 4 & 6 \\
\hline sink & .025 & .250 & .135 & .135 \\
\hline spatula & .025 & .109 & .063 & .202 \\
\hline dishwasher & & .328 & .040 & .048 \\
\hline cup & & .109 & .119 & .202 \\
\hline skillet & & .094 & .016 & .058 \\
\hline coffee pot & & .063 & .032 & \\
\hline measuring cup & & .063 & .032 & .038 \\
\hline table & & .063 & .024 & .029 \\
\hline toaster & & .063 & .024 & .067 \\
\hline broom & & .047 & .079 & .019 \\
\hline can & & .047 & .016 & \\
\hline bottle opener & & .031 & & \\
\hline cabinet & & .031 & & \\
\hline cupboard & & .031 & & \\
\hline dustpan & & .031 & .016 & \\
\hline garbage & & .031 & & \\
\hline mop & & .031 & .024 & .019 \\
\hline pot holder & & .031 & & \\
\hline potato masher & & .031 & & \\
\hline scissors & & .031 & & \\
\hline screwdriver & & .031 & & \\
\hline sponge & & .031 & .024 & .019 \\
\hline tablecloth & & .031 & & \\
\hline bowls & & & .048 & .019 \\
\hline rag & & & .040 & \\
\hline plates & & & .032 & \\
\hline salt & & & .024 & \\
\hline soap & & & .024 & \\
\hline towel & & & .024 & \\
\hline cutter & & & .016 & \\
\hline cutting board & & & .016 & \\
\hline glasses & & & .016 & .019 \\
\hline grater & & & .016 & \\
\hline hammer & & & .016 & \\
\hline meat & & & .016 & \\
\hline napkin & & & .016 & \\
\hline rolling pin & & & .016 & .019 \\
\hline silverware & & & .016 & .029 \\
\hline soup spoon & & & .016 & \\
\hline water & & & .016 & \\
\hline board & & & & .058 \\
\hline disposal & & & & .038 \\
\hline fry pan & & & & .038 \\
\hline light & & & & .029 \\
\hline trashmasher & & & & .029 \\
\hline ladle & & & & .019 \\
\hline pressure cooker & & & & .019 \\
\hline strainer & & & & .019 \\
\hline sugar & & & & .019 \\
\hline trash can & & & & .019 \\
\hline washer & & & & .019 \\
\hline Mean & 3.65 & 7.06 & 5.67 & 6.72 \\
\hline
\end{tabular}

\begin{tabular}{|c|c|c|c|c|}
\hline \multicolumn{5}{|c|}{$\begin{array}{l}\text { Category } 9 \\
\text { Vegetables }\end{array}$} \\
\hline \multirow[b]{2}{*}{ Instance } & \multicolumn{4}{|c|}{ Grade } \\
\hline & 2 & 3 & 4 & 6 \\
\hline $\begin{array}{l}\text { carrot } \\
\text { peas } \\
\text { corn } \\
\text { tomato }\end{array}$ & $\begin{array}{l}.481 \\
.420 \\
.259 \\
.247\end{array}$ & $\begin{array}{l}.766 \\
.547 \\
.484 \\
.391\end{array}$ & $\begin{array}{l}.595 \\
.516 \\
.484 \\
.421\end{array}$ & $\begin{array}{l}.779 \\
.644 \\
.500 \\
.519\end{array}$ \\
\hline
\end{tabular}


Category 9 Continued

\begin{tabular}{lcccc}
\hline \multicolumn{1}{c}{ Instance } & \multicolumn{3}{c}{ Grade } \\
\cline { 2 - 5 } \multicolumn{1}{c}{} & 2 & 3 & 4 & 6 \\
\hline beans & .185 & .453 & .429 & .519 \\
spinach & .173 & .250 & .214 & .413 \\
potato & .148 & .422 & .317 & \\
radish & .148 & .141 & .032 & .115 \\
lettuce & .099 & .281 & .238 & .394 \\
beet & .074 & .063 & .127 & .183 \\
celery & .062 & .156 & .286 & .269 \\
asparagus & .049 & .031 & .024 & .067 \\
cabbage & .037 & .188 & .143 & .154 \\
salad & .037 & & .016 & .019 \\
broccoli & .025 & .109 & & .019 \\
cucumber & .025 & .094 & .032 & .077 \\
green beans & .025 & .094 & .016 & .029 \\
onion & & .125 & .087 & .125 \\
squash & & .094 & .175 & .077 \\
string bean & & .094 & .024 & .048 \\
cauliflower & & .078 & .024 & .048 \\
sauerkraut & & .078 & & \\
lima beans & & .047 & & .038 \\
mixed vegetables & & .031 & & \\
peanut \\
pumpkin
\end{tabular}

\begin{tabular}{|c|c|c|c|c|}
\hline \multicolumn{5}{|c|}{$\begin{array}{l}\text { Category } 10 \\
\text { Sports }\end{array}$} \\
\hline \multirow[b]{2}{*}{ Instance } & \multicolumn{4}{|c|}{ Grade } \\
\hline & 2 & 3 & 4 & 6 \\
\hline football & .852 & .766 & .865 & .942 \\
\hline baseball & .568 & .641 & .778 & .894 \\
\hline basketball & .469 & .469 & .571 & .673 \\
\hline soccer & .309 & .344 & .548 & .615 \\
\hline hockey & .210 & .188 & .246 & .308 \\
\hline kickball & .136 & .281 & .413 & .394 \\
\hline swimming & .099 & .188 & .246 & .317 \\
\hline tennis & .099 & .203 & .127 & .183 \\
\hline dodge ball & .086 & & & .048 \\
\hline golf & .074 & .109 & .056 & .048 \\
\hline jump rope & .074 & .031 & .024 & \\
\hline racing & .062 & .125 & .024 & .019 \\
\hline ball & .037 & & & .019 \\
\hline fishing & .037 & .094 & .032 & \\
\hline volleyball & .037 & .047 & .349 & .279 \\
\hline roller-skating & .037 & .031 & .056 & .019 \\
\hline skating & .025 & & .040 & .067 \\
\hline skiing & & .219 & .119 & .212 \\
\hline Monopoly & & .203 & .016 & .058 \\
\hline Life & & .141 & .040 & .019 \\
\hline softball & & .125 & .071 & .125 \\
\hline bowling & & .094 & .063 & .029 \\
\hline
\end{tabular}

Category 10 Continued

\begin{tabular}{|c|c|c|c|c|}
\hline \multirow[b]{2}{*}{ Instarice } & \multicolumn{4}{|c|}{ Grade } \\
\hline & 2 & 3 & 4 & 6 \\
\hline cards & & .094 & & .019 \\
\hline chess & & .094 & & .019 \\
\hline shuffleboard & & .094 & & \\
\hline hide-and-seek & & .078 & & \\
\hline ice-skating & & .078 & .109 & .019 \\
\hline poker & & .063 & & \\
\hline pool & & .063 & .016 & .058 \\
\hline track & & .063 & .063 & .019 \\
\hline Yahtzee & & .063 & & \\
\hline handball & & .047 & & .038 \\
\hline hopscotch & & .047 & .079 & \\
\hline Ping-Pong & & .047 & .016 & .029 \\
\hline tag & & .047 & .016 & .048 \\
\hline badmitton & & .031 & & .029 \\
\hline boating & & .031 & & \\
\hline boxing & & .031 & .032 & \\
\hline chase & & .031 & & \\
\hline checkers & & .031 & & \\
\hline duck-duck & & .031 & & \\
\hline high jump & & .031 & & \\
\hline hiking & & .031 & & .038 \\
\hline Kerplunk & & .031 & & \\
\hline running & & .031 & .016 & \\
\hline Scrabble & & .031 & & \\
\hline sevenup & & .031 & & \\
\hline foursquare & & & .040 & .183 \\
\hline horseback riding & & & .040 & .029 \\
\hline polo & & & .040 & .029 \\
\hline red rover & & & .040 & .048 \\
\hline tumbling & & & .024 & \\
\hline pinball & & & .016 & \\
\hline riding & & & .016 & \\
\hline roller derby & & & .016 & \\
\hline tetherball & & & .016 & \\
\hline rugby & & & & .048 \\
\hline keep-away & & & & .029 \\
\hline capture flag & & & & .019 \\
\hline hunting & & & & .019 \\
\hline Parcheesi & & & & .019 \\
\hline ticktacktoe & & & & .019 \\
\hline Twister & & & & .019 \\
\hline water polo & & & & .019 \\
\hline water skiing & & & & .019 \\
\hline Mean & 2.89 & 6.94 & 4.54 & 6.47 \\
\hline
\end{tabular}

Category 11

Denominations of Money

\begin{tabular}{lcccc}
\hline & \multicolumn{4}{c}{ Grade } \\
\cline { 2 - 5 } \multicolumn{1}{c}{ Instance } & 2 & 3 & 4 & 6 \\
\hline dollar & .679 & .828 & .746 & .904 \\
dime & .667 & .688 & .603 & .788 \\
penny & .654 & .656 & .667 & .798 \\
nickel & .519 & .578 & .667 & .644 \\
quarter & .383 & .641 & .611 & .760 \\
half dollar & .111 & .484 & .032 & .279 \\
silver dollar & .062 & .141 & & .077 \\
cent & .037 & .094 & .095 & .048 \\
coin & & .047 & & .038 \\
peso & & .047 & & .038 \\
bank & & .031 & & \\
gold & & .031 & .032 &
\end{tabular}


Category 11 Continued

\begin{tabular}{llccc}
\hline & \multicolumn{3}{c}{ Grade } \\
\cline { 2 - 5 } \multicolumn{1}{l}{ Instance } & 2 & 3 & 4 & 6 \\
\hline silver & & .040 & \\
copper & & .024 & \\
counterfeit & & .016 & \\
yen & & & .048 \\
marks & & & .029 \\
pint & & & .019 \\
pound & & & .019 \\
Mean & 3.12 & 4.58 & 3.63 & 4.57 \\
\hline
\end{tabular}

\begin{tabular}{|c|c|c|c|c|}
\hline & & $\begin{array}{l}\text { y } 12 \\
\text { vear }\end{array}$ & & \\
\hline & & & & \\
\hline Instance & 2 & 3 & 4 & 6 \\
\hline socks & .778 & .750 & .698 & .865 \\
\hline shoes & .728 & .797 & .778 & .894 \\
\hline boots & .605 & .547 & .603 & 1.000 \\
\hline slippers & .358 & .438 & .532 & .394 \\
\hline tennis & .222 & .375 & .206 & .327 \\
\hline sandals & .210 & .359 & .262 & .721 \\
\hline moccasins & .111 & .203 & .024 & .067 \\
\hline sneakers & .049 & .063 & .071 & .019 \\
\hline high heels & .037 & .063 & .071 & .029 \\
\hline cowboy boot & .025 & .031 & & \\
\hline high tops & .025 & & & \\
\hline hiking boots & .025 & .094 & & .087 \\
\hline hose & .025 & & .063 & .077 \\
\hline rubbers & .025 & & .024 & .029 \\
\hline saddle shoes & .025 & & .016 & .019 \\
\hline snowshoes & .025 & .031 & & \\
\hline thongs & .025 & .063 & .079 & .192 \\
\hline toe nails & & .125 & & \\
\hline stockings & & .109 & .016 & .096 \\
\hline cleats & & .078 & & \\
\hline overboots & & .078 & & \\
\hline toes & & .078 & & \\
\hline blister & & .063 & & \\
\hline shoelaces & & .063 & & \\
\hline anklet & & .047 & & \\
\hline house slippers & & .047 & & \\
\hline street shoes & & .047 & & \\
\hline track shoes & & .047 & & \\
\hline feet & & .031 & & \\
\hline fins & & .031 & & \\
\hline flippers & & .031 & & .015 \\
\hline gogo boots & & .031 & .016 & \\
\hline golf shoes & & .031 & & \\
\hline hard shoes & & .031 & & \\
\hline ice skates & & .031 & .048 & \\
\hline knee socks & & .031 & & \\
\hline overshoes & & .031 & & \\
\hline roller skates & & .031 & .024 & \\
\hline slipons & & .031 & & \\
\hline toe nail polish & & .031 & & \\
\hline clogs & & & .103 & .15 \\
\hline heels & & & .040 & \\
\hline skates & & & .032 & .02 \\
\hline skis & & & .032 & \\
\hline sleepers & & & .032 & \\
\hline skins & & & .024 & \\
\hline clutter boots & & & .016 & .01 \\
\hline dance shoes & & & .016 & \\
\hline
\end{tabular}

Category 12 Continued

\begin{tabular}{|c|c|c|c|c|}
\hline \multirow[b]{2}{*}{ Instance } & \multicolumn{4}{|c|}{ Grade } \\
\hline & 2 & 3 & 4 & 6 \\
\hline footlets & & & .016 & \\
\hline galoshes & & & .016 & .019 \\
\hline pantyhose & & & .016 & .058 \\
\hline nylons & & & & .087 \\
\hline footsie & & & & .077 \\
\hline Band-Aids & & & & .038 \\
\hline desert boots & & & & .029 \\
\hline football shoes & & & & .029 \\
\hline heel & & & & .029 \\
\hline ski boots & & & & .029 \\
\hline skin & & & & .029 \\
\hline loafers & & & & .019 \\
\hline mud & & & & .019 \\
\hline play shoes & & & & .019 \\
\hline powder & & & & .019 \\
\hline Sunday shoes & & & & .019 \\
\hline tights & & & & .019 \\
\hline waders & & & & .019 \\
\hline Mean & 3.46 & 5.89 & 4.10 & 5.99 \\
\hline
\end{tabular}

\section{Category 13} Toys

\begin{tabular}{|c|c|c|c|c|}
\hline \multirow[b]{2}{*}{ Instance } & \multicolumn{4}{|c|}{ Grade } \\
\hline & 2 & 3 & 4 & 6 \\
\hline doll & .370 & .672 & .540 & .663 \\
\hline ball & .321 & .391 & .270 & .308 \\
\hline car & .259 & .281 & .437 & .471 \\
\hline truck & .210 & .297 & .373 & .394 \\
\hline top & .173 & .172 & .159 & .106 \\
\hline boat & .148 & .063 & .032 & .019 \\
\hline train & .148 & .094 & .103 & .173 \\
\hline airplane & .086 & & .024 & .038 \\
\hline bike & .074 & .141 & .151 & .067 \\
\hline game & .062 & .125 & .183 & .221 \\
\hline teddy bear & .062 & .172 & .016 & \\
\hline bat & .049 & & .048 & .106 \\
\hline roller skates & .049 & & .024 & \\
\hline tractor & .049 & .125 & .111 & \\
\hline Barbie & .037 & .031 & .079 & .106 \\
\hline blocks & .037 & .047 & .032 & .087 \\
\hline Hot Wheels & .037 & .063 & .063 & .019 \\
\hline toy & .037 & & .024 & .029 \\
\hline baseball & .025 & & .056 & .067 \\
\hline bicycle & .025 & .031 & & \\
\hline football & .025 & .078 & .143 & .125 \\
\hline house & .025 & & & .029 \\
\hline putt & .025 & & & \\
\hline stuffed animal & .025 & .078 & .024 & .058 \\
\hline yoyo & .025 & .078 & .079 & \\
\hline puzzle & & .125 & .048 & \\
\hline book & & .078 & .016 & \\
\hline gun & & .078 & .111 & .202 \\
\hline paper doll & & .078 & & \\
\hline rubber duck & & .078 & .024 & .019 \\
\hline $\operatorname{dog}$ & & .063 & .040 & .019 \\
\hline basketball & & .047 & .040 & .058 \\
\hline dish & & .047 & & .058 \\
\hline Lego & & .047 & & \\
\hline model & & .047 & & .087 \\
\hline
\end{tabular}


Category 13 Continued

\begin{tabular}{|c|c|c|c|c|}
\hline \multirow[b]{2}{*}{ Instance } & \multicolumn{4}{|c|}{ Grade } \\
\hline & 2 & 3 & 4 & 6 \\
\hline animal & & .031 & .056 & .106 \\
\hline bars & & .031 & & \\
\hline cat & & .031 & .016 & \\
\hline color book & & .031 & & \\
\hline cowboy & & .031 & & \\
\hline dump truck & & .031 & & \\
\hline Hoppity Hop & & .031 & & \\
\hline horse & & .031 & .016 & .029 \\
\hline jack-in-the-box & & .031 & & \\
\hline Life & & .031 & & \\
\hline logs & & .031 & & \\
\hline minibike & & .031 & & \\
\hline Monopoly & & .031 & & \\
\hline motorcycle & & .031 & & \\
\hline mouse & & .031 & & \\
\hline playground & & .031 & & \\
\hline rattle & & .031 & & .106 \\
\hline record & & .031 & & \\
\hline robot & & .031 & .032 & .019 \\
\hline Sizzlers & & .031 & & \\
\hline swing & & .031 & .032 & \\
\hline wagon & & .031 & .048 & \\
\hline Matchbox & & & .063 & \\
\hline trunk & & & .056 & \\
\hline doll house & & & .048 & .019 \\
\hline GI Joe & & & .032 & .067 \\
\hline jeep & & & .024 & \\
\hline windup & & & .024 & \\
\hline bear & & & .016 & \\
\hline Big Jim & & & .016 & \\
\hline cards & & & .016 & \\
\hline crib & & & .016 & \\
\hline drum & & & .016 & \\
\hline duck & & & .016 & .019 \\
\hline helicopter & & & .016 & \\
\hline jacks & & & .016 & .019 \\
\hline mat & & & .016 & \\
\hline plane & & & .016 & .096 \\
\hline race car & & & .016 & \\
\hline rope & & & .016 & .029 \\
\hline skate & & & .016 & \\
\hline slide & & & .016 & \\
\hline tanks & & & .016 & .019 \\
\hline trampoline & & & .016 & \\
\hline bell & & & & .048 \\
\hline puppet & & & & .038 \\
\hline ships & & & & .038 \\
\hline hopscotch & & & & .029 \\
\hline jump rope & & & & .029 \\
\hline mitt & & & & .029 \\
\hline rocket & & & & .029 \\
\hline soldier & & & & .029 \\
\hline balloons & & & & .019 \\
\hline board & & & & .019 \\
\hline buggy & & & & .019 \\
\hline dingaling & & & & .019 \\
\hline hula hoop & & & & .019 \\
\hline play dough & & & & .019 \\
\hline race & & & & .019 \\
\hline trike & & & & .019 \\
\hline Mean & 3.05 & 5.47 & 4.26 & 5.36 \\
\hline
\end{tabular}

Category 14 Trees

\begin{tabular}{lllll} 
& \multicolumn{4}{c}{ Trees } \\
\cline { 2 - 5 } \multicolumn{1}{c}{ Instance } & \multicolumn{4}{c}{ Grade } \\
\hline & & 3 & 4 & 6 \\
apple & & & & \\
maple & .593 & .547 & .595 & .587 \\
pine & .346 & .297 & .460 & .577 \\
orange & .333 & .438 & .524 & .750 \\
pear & .148 & .281 & .302 & .192 \\
spruce & .123 & .078 & .167 & .221 \\
cherry & .099 & .063 & .095 & .462 \\
green & .074 & .250 & .222 & .240 \\
oak & .074 & .063 & & \\
plum & .074 & .531 & .611 & .683 \\
evergreen & .074 & .203 & .087 & .077 \\
grape & .062 & .094 & .159 & .240 \\
palm & .062 & .047 & .048 & \\
peach & .062 & .078 & .135 & .125 \\
aspen & .062 & .297 & .143 & .183 \\
blue spruce & .049 & .234 & .214 & .231 \\
coconut & .049 & .141 & .024 & .048 \\
pineapple & .049 & .047 & .040 & .019 \\
willow & .049 & & .048 & \\
tree & .049 & .063 & .063 & .212 \\
alpine & .037 & & & \\
banana & .025 & & .019 \\
fruit & .025 & .047 & .079 & .029 \\
& .025 & & &
\end{tabular}

fruit $\quad .025$

honey $\quad .025$

lemon $\quad .025$

red $\quad .025$

leaves

branch

nest

rubber

sap

trunk

.125

.094

.063

.063

.063

bird $\quad .047$

gum $\quad .047$

redwood $\quad .047$

weeping willow $\quad .047$

bark .031

big $\quad .031$

brown .031

cedar

crabapple

little

olive

paper

stump

walnut

elm

fir

birch

Christmas

cottonwood

hollow

nut

dogwood

grapefruit

ash

cotton

apricot

sycamore

chestnut

locust
.079

.029

.024

.016

.024

.219

\section{7}

750

.192

221

.462

.683

.077

.125

.183

048

019

212

19

.063

.230

.115

.016

.031

.031

.031

.031

.031

.031

$\begin{array}{ll}.032 & .019 \\ .151 & .404 \\ .063 & .144 \\ .056 & .144 \\ .040 & .019 \\ .032 & .106 \\ .016 & \\ .016 & \\ & .096 \\ & .058 \\ & .048 \\ & .038 \\ & .029 \\ & .029 \\ & .019 \\ & .019\end{array}$


Category 14 Continued

\begin{tabular}{lcccc} 
& \multicolumn{4}{c}{ Grade } \\
\cline { 2 - 5 } \multicolumn{1}{c}{ Instance } & 2 & 3 & 4 & 6 \\
\hline mulberry & & & & .019 \\
poplar & & & & .019 \\
Mean & 2.86 & 5.66 & 4.98 & 6.69 \\
\hline
\end{tabular}

Category 15
Clothing

\begin{tabular}{|c|c|c|c|c|}
\hline Instance & 2 & 3 & 4 & 6 \\
\hline pants & .667 & .797 & .802 & .837 \\
\hline shirt & .630 & .797 & .762 & .856 \\
\hline dress & .469 & .656 & .540 & .519 \\
\hline socks & .333 & .531 & .484 & .663 \\
\hline shoes & .185 & 438 & .175 & .413 \\
\hline underwear & .173 & .203 & .476 & .625 \\
\hline hat & .136 & .172 & .087 & .183 \\
\hline skirt & .136 & .313 & .175 & .298 \\
\hline shorts & .099 & .266 & .198 & .192 \\
\hline coat & .086 & .203 & .143 & .202 \\
\hline blouse & .074 & .094 & .103 & .337 \\
\hline t-shirt & .074 & .109 & .040 & .096 \\
\hline scarf & .062 & .031 & & .019 \\
\hline sweater & .062 & .047 & & .106 \\
\hline slip & .049 & .031 & .079 & .144 \\
\hline tie & .049 & .109 & .079 & .048 \\
\hline gloves & .037 & .094 & .032 & .048 \\
\hline top & .037 & & & .019 \\
\hline boots & .025 & .047 & .016 & .029 \\
\hline undershirt & .025 & .078 & .048 & .029 \\
\hline vest & & .141 & .087 & .077 \\
\hline hotpants & & .063 & & \\
\hline pajamas & & .063 & .032 & .038 \\
\hline bra & & .047 & .175 & .337 \\
\hline mittens & & .047 & .024 & \\
\hline belt & & .031 & .040 & .125 \\
\hline culottes & & .031 & & \\
\hline flares & & .031 & & \\
\hline jumper & & .031 & .032 & .029 \\
\hline Levis & & .031 & & \\
\hline purse & & .031 & & \\
\hline slacks & & .031 & .040 & .038 \\
\hline sport shirt & & .031 & & \\
\hline stockings & & .031 & & .019 \\
\hline suit & & .031 & .024 & .019 \\
\hline swimsuit & & .031 & & .048 \\
\hline underpants & & .031 & & .058 \\
\hline yarn & & .031 & & \\
\hline girdle & & & .079 & .144 \\
\hline sweatshirt & & & .040 & .038 \\
\hline hose & & & .032 & .125 \\
\hline panties & & & .032 & \\
\hline pantyhose & & & .032 & \\
\hline smock & & & .032 & .019 \\
\hline pantsuit & & & .024 & \\
\hline cutoffs & & & .016 & \\
\hline jacket & & & .016 & .019 \\
\hline jeans & & & .016 & .019 \\
\hline diaper & & & & .067 \\
\hline nylon & & & & .067 \\
\hline bathing suit & & & & .019 \\
\hline bikini & & & & .019 \\
\hline
\end{tabular}

Category 15 Continued

\begin{tabular}{lcccc}
\hline & \multicolumn{4}{c}{ Grade } \\
\cline { 2 - 5 } \multicolumn{1}{l}{ Instance } & 2 & 3 & 4 & 6 \\
\hline bodysuit & & & & .019 \\
jock & & & & .019 \\
nightgown & & & & .019 \\
Mean & 3.72 & 5.98 & 5.22 & 7.34 \\
\hline
\end{tabular}

\begin{tabular}{|c|c|c|c|c|}
\hline \multirow[b]{3}{*}{ Instance } & \multicolumn{2}{|c|}{$\begin{array}{c}\text { Category } 16 \\
\text { Relatives }\end{array}$} & & \\
\hline & \multicolumn{4}{|c|}{ Grade } \\
\hline & 2 & 3 & 4 & 6 \\
\hline aunt & .568 & .781 & .833 & .933 \\
\hline uncle & .543 & .734 & .762 & .952 \\
\hline grandma & .259 & .344 & .167 & .260 \\
\hline cousin & .235 & .578 & .683 & .654 \\
\hline dad & .185 & .203 & .087 & .135 \\
\hline grandpa & .185 & .359 & .135 & .240 \\
\hline mom & .185 & .141 & .071 & .125 \\
\hline brother & .173 & .516 & .286 & .490 \\
\hline sister & .160 & .422 & .302 & .510 \\
\hline mother & .111 & .219 & .270 & .404 \\
\hline grandmother & .099 & .172 & .563 & .519 \\
\hline daddy & .074 & .031 & & \\
\hline baby & .062 & & .016 & \\
\hline father & .062 & .141 & .254 & .375 \\
\hline grandfather & .062 & .188 & .500 & .500 \\
\hline & .049 & .063 & & \\
\hline mommy & .049 & & & \\
\hline grandmom & .037 & .031 & & \\
\hline nephew & .037 & .031 & .071 & .096 \\
\hline granddad & .025 & & & .019 \\
\hline gramma & & .125 & .024 & \\
\hline niece & & .125 & .071 & .163 \\
\hline grampa & & .094 & .024 & .019 \\
\hline great grandma & & .063 & & .038 \\
\hline cat & & .047 & & \\
\hline great gramma & & .047 & & \\
\hline stepfather & & .047 & & .019 \\
\hline stepmother & & .047 & & .019 \\
\hline friends & & .031 & & \\
\hline great aunt & & .031 & & .058 \\
\hline great granddad & & .031 & .016 & \\
\hline great grandmother & & .031 & & \\
\hline great grand pa & & .031 & & .038 \\
\hline great uncle & & .031 & & .029 \\
\hline grandparent & & & .063 & .087 \\
\hline parents & & & & .038 \\
\hline second cousin & & & & .029 \\
\hline great grandfather & & & & .019 \\
\hline neighbor & & & & .019 \\
\hline Mean & 3.44 & 5.47 & 5.25 & 6.95 \\
\hline
\end{tabular}

Category 17

Furniture

\begin{tabular}{lcccc}
\hline & \multicolumn{4}{c}{ Grade } \\
\cline { 2 - 5 } \multicolumn{1}{c}{ Instance } & 2 & 3 & 4 & 6 \\
\hline chair & .790 & .938 & .944 & 1.000 \\
couch & .630 & .672 & .667 & .779 \\
table & .531 & .719 & .786 & .865
\end{tabular}


Category 17 Continued

\begin{tabular}{|c|c|c|c|c|}
\hline \multirow[b]{2}{*}{ Instance } & \multicolumn{4}{|c|}{ Grade } \\
\hline & 2 & 3 & 4 & 6 \\
\hline bed & .272 & .578 & .667 & .798 \\
\hline desk & .198 & .438 & .397 & .510 \\
\hline dresser & .111 & .234 & .270 & .279 \\
\hline sofa & .111 & .250 & .175 & .250 \\
\hline television & .111 & .125 & .095 & .135 \\
\hline lamp & .062 & .156 & .135 & .240 \\
\hline stool & .062 & .094 & .024 & .115 \\
\hline rocker & .049 & .063 & & \\
\hline love seat & .037 & .078 & .087 & .250 \\
\hline shelf & .037 & .031 & .024 & .038 \\
\hline beanbag & .025 & & & .019 \\
\hline bed table & .025 & & & \\
\hline bench & .025 & & .071 & .087 \\
\hline coffee table & .025 & .078 & & .019 \\
\hline stove & & .094 & .016 & .019 \\
\hline rug & & .063 & .024 & \\
\hline cabinet & & .047 & .024 & .077 \\
\hline refrigerator & & .047 & & .019 \\
\hline bookcase & & .031 & & .048 \\
\hline carpet & & .031 & & \\
\hline chest & & .031 & .040 & .029 \\
\hline davenport & & .031 & & \\
\hline piano & & .031 & .032 & \\
\hline rocking chair & & .031 & .016 & \\
\hline sink & & .031 & .024 & .058 \\
\hline stereo & & & .063 & .058 \\
\hline TV desk & & & .056 & \\
\hline TV bed & & & .032 & \\
\hline light & & & .024 & \\
\hline night stand & & & .024 & .038 \\
\hline vanity & & & .024 & \\
\hline bookshelf & & & .016 & .019 \\
\hline $\cot$ & & & .016 & \\
\hline footstool & & & .016 & .019 \\
\hline recorder & & & .016 & \\
\hline water bed & & & .016 & .058 \\
\hline toilet & & & & .087 \\
\hline drawers & & & & .048 \\
\hline mirror & & & & .038 \\
\hline recliner & & & & .038 \\
\hline bar & & & & .029 \\
\hline end table & & & & .029 \\
\hline hassock & & & & .029 \\
\hline tub & & & & .029 \\
\hline arm chair & & & & .019 \\
\hline & & & & .019 \\
\hline bathtub & & & & .019 \\
\hline clock & & & & .019 \\
\hline hutch & & & & .019 \\
\hline Mean & 3.32 & 4.00 & 5.15 & 6.54 \\
\hline
\end{tabular}

\begin{tabular}{lcccc}
\multicolumn{5}{c}{$\begin{array}{c}\text { Category 18 } \\
\text { Crimes }\end{array}$} \\
\cline { 2 - 5 } & \multicolumn{5}{c}{ Grade } \\
\multicolumn{1}{c}{ Instance } & 2 & 3 & 4 & 6 \\
\hline stealing & .580 & .688 & .556 & .462 \\
speeding & .346 & .422 & .452 & .260 \\
killing & .259 & .375 & .476 & .260 \\
robbery & .222 & .281 & .286 & .423 \\
murder & .123 & .531 & .111 & .404 \\
running light & .099 & .094 & & .056
\end{tabular}

Category 18 Continued

\begin{tabular}{|c|c|c|c|c|}
\hline \multirow[b]{2}{*}{ Instance } & \multicolumn{4}{|c|}{ Grade } \\
\hline & 2 & 3 & 4 & 6 \\
\hline shooting & .074 & .156 & .151 & .173 \\
\hline kidnapping & .062 & .078 & .159 & .135 \\
\hline fighting & .049 & & .032 & \\
\hline drugs & .037 & .219 & .143 & .510 \\
\hline breaking & .025 & & & \\
\hline breaking window & .025 & & & .029 \\
\hline jaywalking & .025 & & .048 & .019 \\
\hline rape & .025 & & .032 & .337 \\
\hline crime & & .047 & .016 & .038 \\
\hline drinking & & .047 & .032 & .048 \\
\hline $\sin$ & & .047 & & \\
\hline bombing & & .031 & & \\
\hline driving too slow & & .031 & & \\
\hline handling drugs & & .031 & & \\
\hline littering & & .031 & & \\
\hline shoplifting & & .031 & .032 & .019 \\
\hline stabbing & & .031 & & \\
\hline suicide & & .031 & .032 & .048 \\
\hline wrong parking & & .031 & & \\
\hline racing & & & .048 & \\
\hline burglary & & & .040 & .058 \\
\hline dope & & & .040 & \\
\hline blackmail & & & .024 & .058 \\
\hline drunk & & & .024 & \\
\hline drunk driving & & & .024 & .038 \\
\hline drunkenness & & & .016 & \\
\hline gambling & & & .016 & \\
\hline guns & & & .016 & \\
\hline hitchhiking & & & .016 & .019 \\
\hline theft & & & & .077 \\
\hline felony & & & & .038 \\
\hline adultery & & & & .029 \\
\hline assault & & & & .029 \\
\hline fight & & & & .029 \\
\hline hijacking & & & & .029 \\
\hline manslaughter & & & & .029 \\
\hline smoking pot & & & & .029 \\
\hline arson & & & & .019 \\
\hline battery & & & & .019 \\
\hline counterfeiting & & & & .019 \\
\hline hit-and-run & & & & .029 \\
\hline mugging & & & & .019 \\
\hline trespassing & & & & .019 \\
\hline Mean & 2.31 & 4.33 & 3.04 & 4.17 \\
\hline
\end{tabular}

\begin{tabular}{|c|c|c|c|c|}
\hline \multirow[b]{3}{*}{ Instance } & \multicolumn{2}{|c|}{$\begin{array}{c}\text { Category } 19 \\
\text { Flowers }\end{array}$} & & \\
\hline & \multicolumn{4}{|c|}{ Grade } \\
\hline & 2 & 3 & 4 & 6 \\
\hline rose & .617 & .719 & .825 & .779 \\
\hline daisy & .370 & .578 & .595 & .635 \\
\hline tulip & .321 & .406 & .508 & .558 \\
\hline violet & .284 & .094 & .206 & .212 \\
\hline sunflower & .136 & .094 & .175 & .144 \\
\hline dandelion & .123 & .141 & .143 & .115 \\
\hline forget-me-not & .099 & & & \\
\hline pansy & .099 & & .151 & .163 \\
\hline petunia & .086 & .234 & .056 & .221 \\
\hline daffodil & .074 & .109 & & .135 \\
\hline buttercup & .049 & .047 & .016 & .058 \\
\hline bluebell & .037 & & & .019 \\
\hline
\end{tabular}


Category 19 Continued

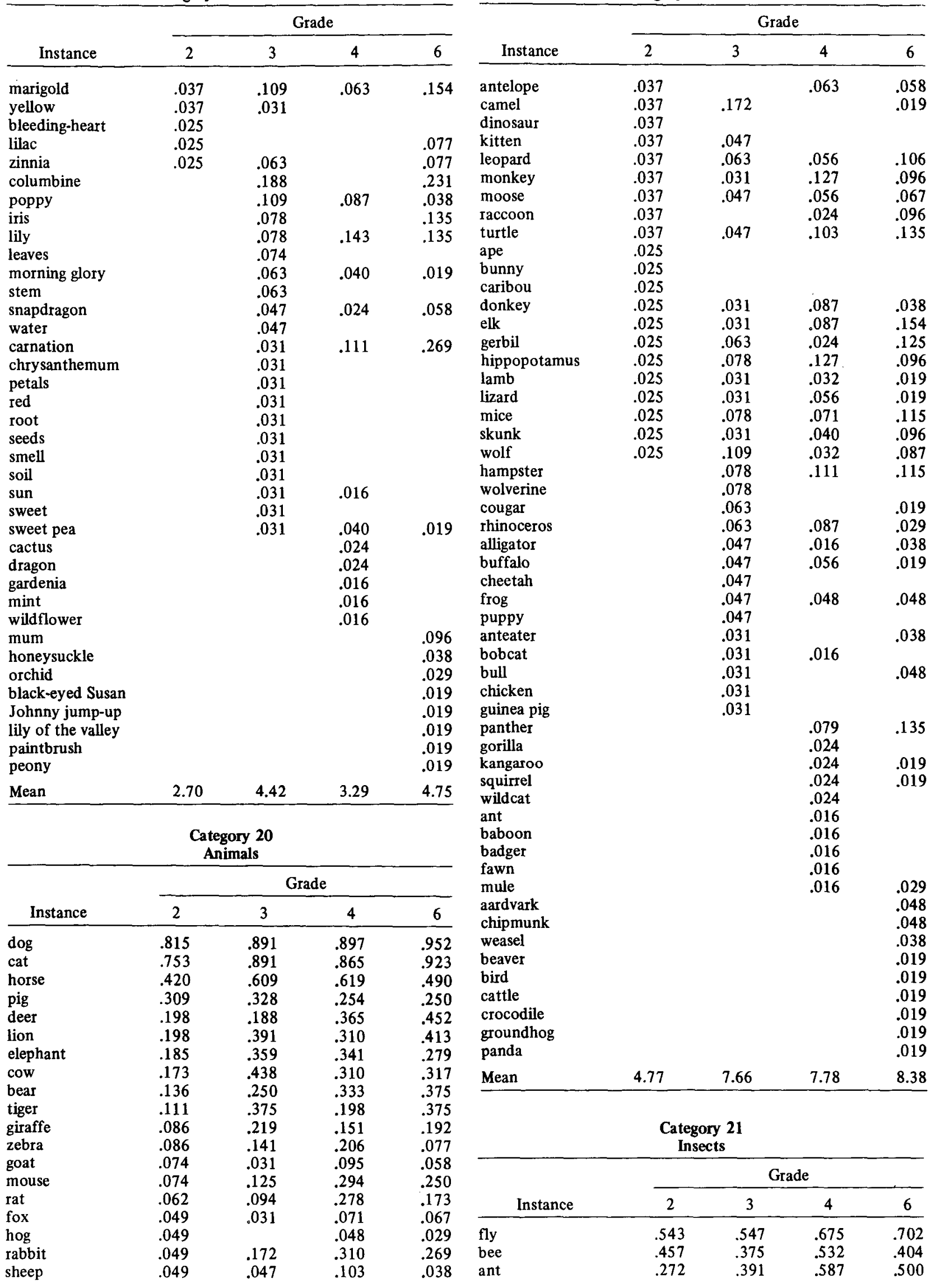

Category 20 Continued 
Category 21 Continued

\begin{tabular}{|c|c|c|c|c|}
\hline \multirow[b]{2}{*}{ Instance } & \multicolumn{4}{|c|}{ Grade } \\
\hline & 2 & 3 & 4 & 6 \\
\hline butterfly & .272 & .266 & .270 & .125 \\
\hline ladybug & .235 & .656 & .262 & .279 \\
\hline spider & .185 & .313 & .444 & .462 \\
\hline black widow spider & .160 & .109 & & \\
\hline mosquito & .160 & .344 & .071 & .385 \\
\hline beetle & .148 & .391 & .389 & .510 \\
\hline grasshopper & .111 & .281 & .429 & .394 \\
\hline caterpillar & .099 & .219 & .032 & .048 \\
\hline worm & .099 & .125 & .143 & .115 \\
\hline tarantula & .074 & .031 & & \\
\hline cricket & .062 & .031 & .167 & .163 \\
\hline bug & .049 & .031 & .024 & .029 \\
\hline tick & .037 & .031 & .024 & .029 \\
\hline wasp & .037 & .078 & .151 & .135 \\
\hline bumblebee & .025 & .031 & & \\
\hline flea & .025 & & .016 & .048 \\
\hline moth & .025 & .063 & .071 & .096 \\
\hline dragonfly & & .078 & .095 & .048 \\
\hline rolly-polly & & .063 & & \\
\hline longlegs & & .031 & & \\
\hline tomato worm & & .031 & & \\
\hline firefly & & & .063 & .029 \\
\hline horsefly & & & .063 & .038 \\
\hline black widow & & & .056 & .087 \\
\hline hornet & & & .032 & .058 \\
\hline gnat & & & .024 & .077 \\
\hline roach & & & .016 & .067 \\
\hline yellowjacket & & & .016 & \\
\hline mantis & & & & .067 \\
\hline stinkbug & & & & .067 \\
\hline cockroach & & & & .048 \\
\hline locust & & & & .048 \\
\hline termite & & & & .038 \\
\hline walking stick & & & & .038 \\
\hline praying mantis & & & & .029 \\
\hline centipede & & & & .019 \\
\hline daddy longlegs & & & & .019 \\
\hline fruit fly & & & & .019 \\
\hline leech & & & & .019 \\
\hline tsetse fly & & & & .019 \\
\hline water bug & & & & .019 \\
\hline Mean & 3.27 & 4.95 & 4.72 & 5.49 \\
\hline
\end{tabular}

\begin{tabular}{lcccc}
\multicolumn{5}{c}{$\begin{array}{c}\text { Category 22 } \\
\text { Colors }\end{array}$} \\
\hline \multicolumn{4}{c}{$\begin{array}{c}\text { Grade } \\
\text { Instance }\end{array}$} & \multicolumn{4}{c}{} \\
\hline red & 2 & 3 & 4 & 6 \\
blue & .889 & .922 & .897 & .962 \\
black & .827 & .953 & .889 & .913 \\
yellow & .617 & .859 & .817 & .962 \\
green & .617 & .734 & .802 & .856 \\
orange & .580 & .750 & .817 & .904 \\
purple & .469 & .594 & .770 & .760 \\
white & .432 & .734 & .714 & .846 \\
brown & .420 & .547 & .635 & .779 \\
pink & .407 & .594 & .611 & .644 \\
gray & .383 & .516 & .484 & .587 \\
gold & .235 & .234 & .357 & .317 \\
silver & .160 & .328 & .460 & .481 \\
& .123 & .297 & .357 & .423
\end{tabular}

Category 22 Continued

\begin{tabular}{lcccc}
\hline & \multicolumn{4}{c}{ Grade } \\
\cline { 2 - 5 } Instance & 2 & 3 & 4 & 6 \\
\hline violet & .062 & .156 & .262 & .135 \\
maroon & .025 & .078 & .024 & .096 \\
orange-red & .025 & & & \\
copper & & .141 & .071 & .096 \\
turquoise & & .063 & .032 & .038 \\
peach & & .047 & .056 & .058 \\
tan & & .047 & .206 & .125 \\
blue-green & & .031 & & .029 \\
mixed & & .031 & & \\
red-orange & & .031 & & \\
red-violet & .031 & & .067 \\
olive & & & .048 & .077 \\
aqua & & & .040 & .087 \\
lavender & & & .032 & .032 \\
salmon & & & .016 & .019 \\
rose & & & & .038 \\
navy & & & .019 \\
hot pink & & & & .019 \\
wine & & & & \\
Mean & 6.43 & & & \\
\hline
\end{tabular}

Category 23

Vehicles and Means of Transportation

\begin{tabular}{|c|c|c|c|c|}
\hline \multirow[b]{2}{*}{ Instance } & \multicolumn{4}{|c|}{ Grade } \\
\hline & 2 & 3 & 4 & 6 \\
\hline car & .914 & 1.000 & .944 & .990 \\
\hline truck & .432 & .484 & .619 & .433 \\
\hline bus & .395 & .547 & .627 & .337 \\
\hline bike & .370 & .328 & .643 & .683 \\
\hline train & .247 & .563 & .444 & .490 \\
\hline airplane & .235 & .328 & .262 & .115 \\
\hline boat & .173 & .203 & .198 & .212 \\
\hline horse & .160 & .156 & .310 & .163 \\
\hline motorcycle & .148 & .297 & .079 & .250 \\
\hline wagon & .123 & .188 & .238 & .183 \\
\hline plane & .099 & .234 & .278 & .404 \\
\hline tractor & .086 & .141 & .071 & .048 \\
\hline bicycle & .049 & .094 & & \\
\hline helicopter & .037 & .078 & .095 & .067 \\
\hline spaceship & .037 & & & \\
\hline taxi & .037 & .125 & .111 & .077 \\
\hline tricycle & .037 & .031 & & \\
\hline buggy & .025 & .063 & .040 & .058 \\
\hline hotrod & .025 & & & \\
\hline rocket & .025 & & .024 & .067 \\
\hline go-cart & & .156 & .048 & .135 \\
\hline jet & & .156 & .079 & .067 \\
\hline dune-buggy & & .094 & & .038 \\
\hline minibike & & .094 & .032 & .096 \\
\hline fire engine & & .078 & & \\
\hline fire truck & & .078 & & \\
\hline jeep & & .063 & .071 & .077 \\
\hline trailer & & .063 & & .067 \\
\hline trike & & .063 & .175 & .269 \\
\hline ship & & .047 & .087 & .029 \\
\hline subway & & .047 & .095 & .058 \\
\hline Big Wheel & & .031 & .024 & .019 \\
\hline camel & & .031 & .024 & \\
\hline feet & & .031 & .040 & \\
\hline ferris wheel & & .031 & & \\
\hline
\end{tabular}


Category 23 Continued

\begin{tabular}{|c|c|c|c|c|}
\hline \multirow[b]{2}{*}{ Instance } & \multicolumn{4}{|c|}{ Grade } \\
\hline & 2 & 3 & 4 & 6 \\
\hline roller coaster & & .031 & & \\
\hline rowboat & & .031 & & \\
\hline unicycle & & .031 & & \\
\hline cycle & & & .246 & .231 \\
\hline camper & & & .079 & .096 \\
\hline $\mathrm{cab}$ & & & .056 & .029 \\
\hline cart & & & .056 & .115 \\
\hline scooter & & & .056 & .038 \\
\hline $\operatorname{tank}$ & & & .040 & .029 \\
\hline donkey & & & .032 & \\
\hline trolley car & & & .032 & .019 \\
\hline balloon & & & .024 & .019 \\
\hline canoe & & & .024 & \\
\hline motorbike & & & .024 & .029 \\
\hline skates & & & .024 & \\
\hline cow & & & .016 & \\
\hline dragster & & & .016 & .019 \\
\hline sled & & & .016 & \\
\hline carriage & & & & .029 \\
\hline chariot & & & & .029 \\
\hline submarine & & & & .029 \\
\hline missile & & & & .019 \\
\hline streetcar & & & & .019 \\
\hline Mean & 4.05 & 6.67 & 6.53 & 6.59 \\
\hline
\end{tabular}

Category 24

Ways to Measure Distance

\begin{tabular}{|c|c|c|c|c|}
\hline \multirow[b]{2}{*}{ Instance } & \multicolumn{4}{|c|}{ Grade } \\
\hline & 2 & 3 & 4 & 6 \\
\hline ruler & .457 & 609 & .627 & .39 \\
\hline yardstick & .333 & .328 & .270 & .11 \\
\hline foot & .284 & .703 & .516 & .75 \\
\hline inch & .148 & .516 & .437 & .67 \\
\hline mile & .123 & .266 & .333 & .58 \\
\hline measuring tape & .086 & & & .01 \\
\hline tape measure & .086 & .156 & & .08 \\
\hline inchworm & .062 & .031 & & \\
\hline tape & .037 & .063 & .190 & .06 \\
\hline yard & .037 & .406 & .373 & .49 \\
\hline footstick & .025 & & & \\
\hline map & .025 & .031 & & \\
\hline string & .025 & .047 & .032 & .02 \\
\hline centimeter & & .250 & .032 & .07 \\
\hline long & & .141 & & \\
\hline short & & .125 & & \\
\hline body & & .078 & & \\
\hline meter & & .078 & .032 & .09 \\
\hline stick & & .063 & & .03 \\
\hline cup & & .047 & & \\
\hline kilometer & & .047 & & .03 \\
\hline meter stick & & .047 & & \\
\hline finger & & .031 & & \\
\hline hand & & .031 & .032 & \\
\hline middle & & .031 & & \\
\hline scale & & .031 & .024 & \\
\hline compass & & & .032 & \\
\hline acres & & & .016 & .0 \\
\hline block & & & .016 & \\
\hline speedometer & & & .016 & \\
\hline
\end{tabular}

Category 24 Continued

\begin{tabular}{|c|c|c|c|c|}
\hline \multirow[b]{2}{*}{ Instance } & \multicolumn{4}{|c|}{ Grade } \\
\hline & 2 & 3 & 4 & 6 \\
\hline year & & & .016 & \\
\hline millimeter & & & & .087 \\
\hline inches & & & & .077 \\
\hline width & & & & .077 \\
\hline fathom & & & & .058 \\
\hline length & & & & .048 \\
\hline league & & & & .038 \\
\hline rod & & & & .038 \\
\hline book & & & & .029 \\
\hline knot & & & & .019 \\
\hline measurer & & & & .019 \\
\hline protracter & & & & .019 \\
\hline Mean & 1.81 & 4.72 & 3.15 & 4.50 \\
\hline
\end{tabular}

Category 25

Ways to Measure Time

\begin{tabular}{lcccc}
\hline & \multicolumn{3}{c}{ Grade } \\
\cline { 2 - 5 } \multicolumn{1}{l}{ Instance } & 2 & 3 & 4 & 6 \\
\hline clock & .481 & .641 & .817 & .192 \\
watch & .370 & .328 & .563 & .096 \\
year & .370 & .375 & .452 & .808 \\
day & .198 & .484 & .429 & .817 \\
week & .173 & .375 & .278 & .529 \\
hour & .111 & .531 & .452 & .692 \\
minute & .099 & .453 & .421 & .740 \\
month & .099 & .406 & .325 & .702 \\
second & .049 & .234 & .294 & .673 \\
radio & .037 & & & \\
sun & .037 & .063 & .143 & .038 \\
hourglass & .025 & & .032 & .038 \\
moon & .025 & & .048 & \\
sundial & .025 & & .063 & .058 \\
time & .025 & .063 & .016 & .019 \\
timer & .025 & & .071 & \\
calendar & & .219 & .040 & .125 \\
number & & .109 & & .029 \\
half-hour \\
century
\end{tabular}

(Received for publication April 11, 1978; revision accepted June 19,1978 .) 